\title{
National Institute of Dental and Craniofacial Research
}

National Cancer Institute

\section{Source}

National Cancer Institute. National Institute of Dental and Craniofacial Research. NCI

Thesaurus. Code C19755.

One of the institutes that comprise the National Institutes of Health. The mission of the NIDCR is to improve oral, dental and craniofacial health through research, research training, and the dissemination of health information. 